\title{
Low back and neck and shoulder pain in members and non-members of adolescents' sports clubs: the Finnish Health Promoting Sports Club (FHPSC) study
}

\author{
M. Rossi ${ }^{1 *}$, K. Pasanen ${ }^{1,8}$, S. Kokko ${ }^{5}$, L. Alanko², O. J. Heinonen ${ }^{3}$, R. Korpelainen ${ }^{4,9,10}$, K. Savonen ${ }^{6}$, H. Selänne , \\ T. Vasankari ${ }^{8}$, L. Kannas ${ }^{5}$, U. Kujala ${ }^{5}$, J. Villberg ${ }^{5}$ and J. Parkkari, ${ }^{1,8}$
}

\begin{abstract}
Background: The objective of this study was to investigate the prevalence of self-reported low back pain (LBP) and neck and shoulder pain (NSP), and the related factors in members and non-members of adolescents' sports clubs.

Methods: This cross-sectional study was based on surveys of 14-16-year-olds as a part of the Finnish Health Promoting Sports Club (FHPSC) Study. The surveys on self-reported health behaviours, injuries, and musculoskeletal health were conducted among sports club members $(n=962)$ and non-members $(n=675)$. Binary logistic regression analysis was applied to study the associations between dependent variables of LBP and NSP, and the independent factors.

Results: The prevalence of LBP during the preceding 3 months was $35.0 \%$ in girls and $24.5 \%$ in boys $(p<0.05$ for sex difference). The prevalence of NSP was $55.9 \%$ in girls and $27.3 \%$ in boys ( $p<0.001$ for sex difference). Being a sports club member increased the odds for LBP in boys (odds ratio [OR] 2.35, $95 \% \mathrm{Cl} 1.48-3.72$ ). On the other hand, sports club participation was associated with lower odds of frequent NSP in girls (OR 0.52, $95 \% \mathrm{Cl}$ 0.33-0.82). No associations were found between other leisure-time physical activity and LBP or NSP. Higher screen time (computer games, TV/DVD, phone, Internet) during leisure-time increased the odds of NSP in boys and LBP in boys and girls.

Conclusions: In this study, self-reported LBP and NSP were already relatively common among adolescents. Girls have a higher risk for reporting LBP and NSP. Measures that are more effective in the prevention of LBP in male sports club members are needed. Excessive screen time is weakly associated with LBP and NSP, which should be taken into account in health promotion among adolescents.
\end{abstract}

Keywords: Neck and shoulder pain, Low back pain, Adolescence, Sports club participation, Prevalence

\section{Background}

Back problems are a major public health problem. In Finland in 2013, back diseases were responsible for a sickness benefit expenditure of approximately 118 million euros, and they caused over two million days of covered illness [1]. Backache itself caused approximately 787,000 covered days of illness [1]. Low back pain (LBP)

\footnotetext{
* Correspondence: marleena.k.rossi@student.jyu.fi

'Tampere Research Center of Sports Medicine, P.O. Box 30FI-33501 Tampere, Finland

Full list of author information is available at the end of the article
}

is relatively common already among adolescents [2]. Neck and shoulder pain (NSP) has been studied less, especially among adolescent athletes [2-4], but the prevalence of NSP seems to have increased during the $21^{\text {st }}$ century [3]. The prevalence of LBP increases with age $[5,6]$. Among 15-16-years-olds, LBP prevalence has been reported to be $32 \%$ in boys and $45 \%$ in girls [7]. Five per cent of those aged 15-16 years $(n=7344)$ sought medical assistance due to their LBP symptoms [7]. LBP in adolescence has a tendency of increasing the probability of LBP also in adulthood [8], and it is commonly concurrent with other 
musculoskeletal pain [9]. Therefore, it is important to identify risk populations and to effect the early prevention of LBP and NSP. Some studies have already investigated the differences in LBP between adolescent athletes and non-athletes $[10,11]$. Physical activity as a risk factor has been studied previously [10,12-14]. However, the results remain inconclusive.

This study is a part of a multidisciplinary and multiinstitutional study (the Finnish Health Promoting Sports Club (FHPSC)) [15] where the overall aim is to investigate the effects of sports club participation and the activity of health promotion within sports clubs on adolescent health. Therefore, the specific objectives of this study were to determine the prevalence, frequency, and severity of LBP and NSP in the 14-16-year-old population. We also explored the associations between LBP and NSP with the health and health behaviour of adolescents, paying special attention to participation in organized sports (sports club membership).

\section{Methods}

This study is part of the Finnish Health Promoting Sports Club (FHPSC) study conducted in Finland by the University of Jyväskylä in conjunction with six sports medicine centres and the UKK institute [15]. This cross-sectional study was based on surveys among 14-16-year-olds, and it was carried out in accordance with the Declaration of Helsinki. The adolescents were notified that they have a right to refuse to participate and withdraw their consent later without giving a reason. A written consent from both a guardian and the adolescent him/herself for the pre- participation screening were obtained for participants under the age of 16. Ethical approval was received from the Ethics Committee of Health Care District of Central Finland (record number 23U/2012). All permission papers included detailed information of the study.

\section{Data collection}

In order to obtain a nationally representative sample of the most popular sports for youths, a total of two hundred and forty youth sports clubs from the ten most popular sports disciplines in Finland (basketball, cross-country skiing, floorball, football, gymnastics, ice-hockey, orienteering, skating, swimming, and track and field) were targeted. Twenty-four clubs were selected from each sport for the sample and 154 youth sports clubs out of 240 participated $(64 \%)$ in the FHPSC study. Data was collected in the middle of the main competition season from January to May 2013 for winter sports, and from August to December 2013 for summer sports. In total, 1889 sports club participants were invited to participate in two separate internet questionnaires (Fig. 1.). From the sports clubs 609 adolescents completed both questionnaires.

In order to compare the health behaviours and health status of youths participating in organized sports clubs (club members) to non-participating youths (non-members), the second sample included in this study was a group of secondary school children aged 14-16. The schools were collated from each district where the sports medicine centres were located, including nearby rural areas. School-based data was collected in two portions following the sports clubs' data collection timeframe (100 schools participated).

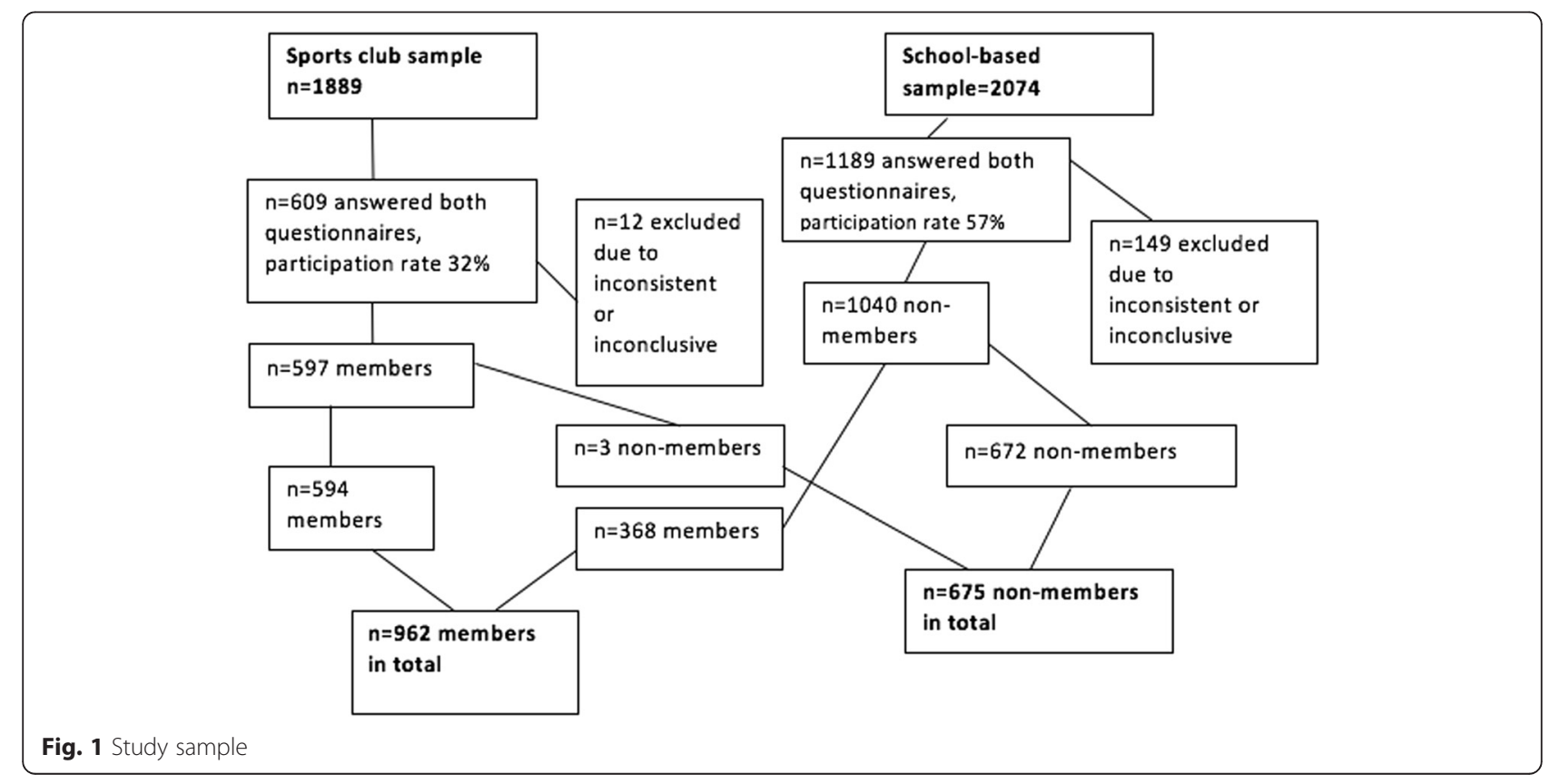


In total, 2074 pupils were asked to participate in the study during the normal school day and 1189 completed both questionnaires.

Members of the school-based sample were asked about their sports club participation ("At the moment are you a member of a sports club?" "no/yes/yes, but I don't participate to training provided by the club") and those who reported being members of a sports club were treated as sports club members $(n=368)$ in the following analyses. Three subjects from the sports club sample were analysed as non-members as they reported not participating in sports club activities (answered no to the question "Are you participating in sports club activities?" "yes/no"). Subjects that provided inconsistent or inconclusive concerning gender and/or age or sports club membership were excluded ( $n=12$ from sports club sample and $n=149$ from schoolbased sample). In total, 962 sports club members (368 from school-based sample and 594 from sports club sample) and 675 non-members were included $(n=1637)$ (Fig. 1.).

\section{Surveys}

Two surveys were conducted (see Additional files 1 and 2). The first focused on the health behaviours of the adolescents, including self-evaluated overall physical activity. The questions included for example: "How many hours on a regular school day you spend your time sitting with one of the following devices? (TV, video/DVD, computer, console games, tablet/phone)" and "Outside school hours: How many hours do you usually do physical activity so that you sweat and get out of breath?". Unlike the questionnaire for non-members, the questionnaire for sports club members included some extra questions on training characteristics, such as active playing/practicing years (at least 2 times a week), training frequency, duration and number of rest days during training and competition seasons, as well as number of competitions. The second questionnaire focused on injuries and the musculoskeletal health of the adolescents. The questions used in these questionnaires were compiled from previously validated questions in other studies, like the Health Behaviour in School-aged Children (HBSC) study [16-20].

\section{Outcomes}

The main dependent outcomes were NSP and LBP within the preceding 3 months. The questions in the questionnaire were "How often have you had the following symptoms in the preceding 3 months?" Answer options included "aches or pain in the neck and shoulders" and "aches or pain in the low back" daily, more than once a week, approximately once a week, 2-3 times a month, approximately once a month, and less than once a month or not at all. Two dependent variables were formed for both LBP and NSP. These were LBP (low back pain more than once a month) and frequent LBP (low back pain at least once a week), and NSP (neck and shoulder pain more than once a month) and frequent NSP (neck and shoulder pain at least once a week). Questions that were more specifically about LBP were based on the standardized Nordic questionnaire of musculoskeletal symptoms [21]. LBP was defined as "an ache, pain, or discomfort of the lumbar region with or without radiation to one or both legs (sciatica)." The questions in the questionnaire included:

- "Have you ever experienced problems in your low back?" (area illustrated by a picture) (no/yes)

- "Have you ever had surgery because of LBP?" (no/yes)

- "Have you ever had radiating LBP?" (no/yes)

- "Have you ever had sleeping difficulties because of LBP?"(no/yes, how often?)

- "Have you had LBP during the previous 7 days?" (no/yes)

- "Have you experienced low back pain that has required consultation or treatments by a physician, physiotherapist, or chiropractor in the previous 12 months?" (no/yes)

- "How did your LBP symptoms occur?" suddenly (after an injury)/gradually (without a sudden injury)/or both

- "Have you used pain killers (NSAID) for your low back?" (no/yes)

\section{Statistical methods}

IBM SPSS Statistics (v. 22.0) was used to carry out all analyses. Sample size was power calculated by Stata 11.0 using data of Kokko et al. [18]. Differences between the groups were assessed using crosstabs and the chi-square test (and $t$-test when appropriate). The subject characteristics are presented for girls and boys, and sports club members and non-members separately as means \pm SDs and percentages. Low back pain and neck and shoulder pain prevalence are expressed as a number and percentage of members and non-members separately for girls and boys. As multilevel modelling failed to give additional information, binary logistic regression analysis was applied to study the associations between the dependent variables of LBP (low back pain) and NSP (neck and shoulder pain) and the independent factors. Binary logistic regression analyses were adjusted by age, sex, BMI, chronic diseases, smoking, and school attainment level (i.e. school grade average). The binary logistic regression analyses were conducted separately for health, health behaviour and training variables. In the analyses for the health and health behaviour the variables were entered into the model simultaneously. In the analyses for training variables separate analyses were conducted for all variables. Odds ratios are reported with $95 \%$ confidence intervals. $P$-value, 0.05 was regarded significant.

\section{Results}

The significant differences in background characteristics between sports club members and non-members are 
highlighted in Table 1. There were more girls who had already had menarche among non-members than members $(97.5 \%$ vs $92.7 \%, p<0.001)$. The use of dietary supplements and pain killers (NSAIDs) was more frequent among sports club members. They were physically more active in their leisure time than non-members and had shorter daily screen time ((mean) 4.1 vs $5.9 \mathrm{~h} /$ day, $p<0.001)$.

\section{Low back pain}

The prevalence of self-reported LBP during the preceding 3 months was $35.0 \%$ in all girls $(n=865)$ and $24.5 \%$ in all boys $(n=772)(p<0.001$ for sex difference) girls being more likely to have frequent LBP than boys (OR $2.3395 \%$ CI 1.58-3.45). No differences between sports club members and non-members were found in girls for LBP (Table 2). However, the prevalence of LBP during the preceding 3 months was significantly higher in male sports club members than in non-members $(28.1 \%$ vs $18.1 \%, p<0.02$ ) (Table 2).

Among boys, sports club members sought medical assistance due to their LBP significantly more often than non-members did ( $25.9 \%$ vs $5.7 \%$ respectively, $p<0.001)$. They also used significantly more NSAIDs due to LBP (Table 3). Among girls, non-members had more sleeping difficulties due to LBP compared to members (11.6\% vs $17.9 \%, p<0.05$ ) (Table 3). However, LBP that radiated to the lower extremities was more common in female sports club members than in non-members $(23.2 \%$ vs $15.0 \%, p<0.05)$.

\section{Neck and shoulder pain}

The prevalence of self-reported NSP was higher in girls $(52.9 \%)$ than in boys $(27.3 \%)(p<0.001$ for sex difference). In addition, the prevalence of frequent NSP was higher in girls than in boys $(19.8 \%$ vs $5.4 \%, p<0.001$ for sex difference). Girls were more likely to have frequent NSP than boys (OR $4.4495 \%$ CI 3.08-6.40). As shown in Table 4, among girls, non-members had a higher prevalence of NSP than sports club members ( $59.9 \%$ vs $47.1 \%, p<0.001)$. The prevalence of frequent NSP during the preceding 3 months was higher in nonmembers for both girls and boys (Table 4).

\section{Risk factors for low back pain}

Adjusted odds ratios regarding health (Table 5), health behaviour (Table 6), and training characteristics (Table 7) are shown in the tables. LBP was associated with reporting neck, thoracic spine, and lower limb pain in boys and girls, and it was also associated with upper limb pain in boys. Higher screen time, as calculated per additional hour of screen time (computer games, TV/DVD, phone, Internet) during leisure time, increased the odds slightly for LBP in boys (OR 1.07, $95 \%$ CI 1.01-1.12) and girls (OR 1.06, 95 \% CI 1.01-1.10, Table 6). For girls,

Table 1 Subject characteristics by sports club participation and gender

\begin{tabular}{|c|c|c|c|c|c|c|c|c|c|}
\hline \multirow[b]{2}{*}{ Variable } & \multicolumn{3}{|c|}{ Boys $(n=772)$} & \multicolumn{3}{|c|}{ Girls $(n=865)$} & \multicolumn{3}{|c|}{ Total $(n=1637)$} \\
\hline & Member & Non-member & $P$-value & Member & Non-member & $P$-value ${ }^{*}$ & Member & Non-member & $P$-value ${ }^{*}$ \\
\hline Age, mean (SD) & $15.5(1)$ & $15.5(0)$ & 0.643 & $15.5(1)$ & $15.5(0)$ & 0.880 & $15.5(1)$ & $15.5(1)$ & 0.839 \\
\hline BMI, mean (SD) & $20.9(2)$ & 21.5(4) & $<0.05$ & $20.6(2)$ & $21.1(4)$ & $<0.05$ & $20.7(2)$ & $21.3(4)$ & $<0.002$ \\
\hline Menarche, $\%(n=882)$ & - & - & & $92.8 \%$ & $97.5 \%$ & $<0.001$ & - & - & - \\
\hline Chronic disease, ${ }^{\mathrm{a}} \%$ & $30.4 \%$ & $26.4 \%$ & 0.242 & $30.1 \%$ & $29.4 \%$ & 0.818 & $30.2 \%$ & $28.1 \%$ & 0.358 \\
\hline Regular medication, ${ }^{\mathrm{b}} \%$ & $23.5 \%$ & $18.6 \%$ & 0.113 & $29.0 \%$ & $33.2 \%$ & 0.189 & $26.2 \%$ & $27.1 \%$ & 0.680 \\
\hline NSAID use, previous month,\% & $59.6 \%$ & $46.1 \%$ & $<0.001$ & $75.0 \%$ & $73.7 \%$ & 0.655 & $67.2 \%$ & $62.2 \%$ & $<0.05$ \\
\hline Special diet, ${ }^{\mathrm{C}} \%$ & $8.0 \%$ & $5.7 \%$ & 0.245 & $17.2 \%$ & $18.0 \%$ & 0.754 & $12.5 \%$ & $12.9 \%$ & 0.804 \\
\hline Dietary supplements use, ${ }^{d} \%$ & $67.3 \%$ & $36.8 \%$ & $<0.001$ & $70.1 \%$ & $57.2 \%$ & $<0.001$ & $68.7 \%$ & $48.7 \%$ & $<0.001$ \\
\hline No Smoking, \% & $94.5 \%$ & $81.4 \%$ & $<0.001$ & $92.6 \%$ & $73.9 \%$ & $<0.001$ & $93.6 \%$ & $77.0 \%$ & $<0.001$ \\
\hline Screen time, ${ }^{e}$ mean (SD) & $4.6(4)$ & $6.4(5)$ & $<0.001$ & $3.6(2)$ & $5.6(5)$ & $<0.001$ & $4.1(3)$ & $5.9(5)$ & $<0.001$ \\
\hline Leisure time PA, f, g \% & & & $<0.001$ & & & $<0.001$ & & & $<0.001$ \\
\hline Approx. $<30$ min/week & $0.8 \%$ & $15.4 \%$ & & $0.9 \%$ & $18.5 \%$ & & $0.8 \%$ & $17.2 \%$ & \\
\hline Approx. 1-3 h/week & $16.3 \%$ & $53.9 \%$ & & $14.9 \%$ & $56.9 \%$ & & $15.6 \%$ & $55.6 \%$ & \\
\hline Approx. 4-6 h/week or more & $82.9 \%$ & $30.7 \%$ & & $84.2 \%$ & $24.6 \%$ & & $83.5 \%$ & $27.2 \%$ & \\
\hline
\end{tabular}

Statistically significant findings are indicated in bold

* $p$-value for difference between members and non-members of sports clubs

${ }^{a}$ Allergy, asthma, diabetes, epilepsy, heart condition, etc.

${ }^{b}$ Contraceptives or other hormonal medication, allergy, asthma, insulin, epilepsy, or heart or blood pressure medication

'Vegetarian, low carb, lactose free, dairy free, gluten free, or other special diet

${ }^{\mathrm{d}}$ For example, vitamins, protein supplements, amino acid supplements, creatine

'TV, computer, computer/console games, phone, tablet use

fBoys $n=770$, girls $n=863$, total $n=1633$

Intensity: breathlessness and sweating 
Table 2 Prevalence of LBP in members and non-members of sports clubs

\begin{tabular}{|c|c|c|c|c|c|c|c|c|c|c|c|}
\hline \multirow{3}{*}{ Variable } & \multirow{3}{*}{ Category } & \multicolumn{4}{|c|}{ Boys $(n=772)$} & \multirow{3}{*}{$P$-value ${ }^{*}$} & \multicolumn{5}{|c|}{ Girls $(n=865)$} \\
\hline & & \multicolumn{2}{|c|}{ Member } & \multicolumn{2}{|c|}{ Non-member } & & \multicolumn{2}{|c|}{ Member } & \multicolumn{2}{|c|}{ Non-member } & \multirow[t]{2}{*}{$P$-value } \\
\hline & & $n$ & $\%$ & $n$ & $\%$ & & $n$ & $\%$ & $n$ & $\%$ & \\
\hline \multirow[t]{2}{*}{ Lifetime prevalence } & Yes & 259 & 52.7 & 122 & 43.4 & \multirow[t]{2}{*}{$<0.02$} & 284 & 60.3 & 246 & 62.4 & \multirow[t]{2}{*}{0.520} \\
\hline & No & 232 & 47.3 & 159 & 56.6 & & 187 & 39.7 & 148 & 37.6 & \\
\hline \multirow[t]{2}{*}{$\operatorname{LBP}^{\mathrm{a}}$} & Yes & 138 & 28.1 & 51 & 18.1 & \multirow[t]{2}{*}{0.02} & 160 & 34.0 & 143 & 36.3 & \multirow[t]{2}{*}{0.475} \\
\hline & No & 353 & 71.9 & 230 & 81.9 & & 311 & 66.0 & 251 & 63.7 & \\
\hline \multirow[t]{2}{*}{ Frequent $L B P^{b}$} & Yes & 30 & 6.1 & 12 & 4.3 & \multirow[t]{2}{*}{0.278} & 51 & 10.8 & 44 & 11.2 & \multirow[t]{2}{*}{0.874} \\
\hline & No & 461 & 93.9 & 269 & 95.7 & & 420 & 89.2 & 350 & 88.8 & \\
\hline \multirow[t]{2}{*}{ LBP during the last seven days ${ }^{c}$} & Yes & 100 & 38.6 & 39 & 32.0 & \multirow[t]{2}{*}{0.209} & 123 & 43.3 & 110 & 44.7 & \multirow[t]{2}{*}{0.745} \\
\hline & No & 159 & 61.4 & 83 & 68.0 & & 161 & 56.7 & 136 & 55.3 & \\
\hline
\end{tabular}

Statistically significant results are indicated in bold

${ }^{*} p$-value for difference between members and non-members of sports clubs

aLBP more than once a month

${ }^{b}$ LBP at least once a week

'Boys $n=381$, girls $n=530$

screen time exceeding $4 \mathrm{~h}$ /day increased the odds for LBP by 1.46 (95\% CI 1.08-1.95). Associations were not found between leisure-time physical activity and LBP or frequent LBP (Table 6). However, in boys, sports club membership was associated with LBP (OR 2.35, 95 \% CI 1.48-3.72). Furthermore, the odds for frequent LBP was higher in male sports club members than in nonmembers (OR 2.7395 \% CI 1.17-6.34) (Table 6). LBP was associated with smoking in both boys and girls, and with alcohol use in boys (Table 6).

Among boys, the training hours during the training season, the number of competitions/games during the preceding 12 months increased the odds of having LBP as calculated per additional hour of training (Table 7). More rest days during the competition season decreased the odds of having LBP in boys and girls, and more rest

Table 3 Characteristics of LBP in members and non-members of sports clubs

\begin{tabular}{|c|c|c|c|c|c|c|c|c|c|c|c|}
\hline \multirow{3}{*}{ Variables } & \multirow{3}{*}{ Category } & \multicolumn{5}{|c|}{ Boys $(n=381)$} & \multicolumn{5}{|c|}{ Girls $(n=530)$} \\
\hline & & \multicolumn{2}{|c|}{ Member } & \multicolumn{2}{|c|}{ Non-member } & \multirow[t]{2}{*}{$P$-value ${ }^{*}$} & \multicolumn{2}{|c|}{ Member } & \multicolumn{2}{|c|}{ Non-member } & \multirow[t]{2}{*}{$P$-value ${ }^{*}$} \\
\hline & & $n$ & $\%$ & $n$ & $\%$ & & $n$ & $\%$ & $n$ & $\%$ & \\
\hline \multirow{2}{*}{$\begin{array}{l}\text { LBP that has demanded medical } \\
\text { assistance in the previous } 12 \text { months }{ }^{\text {a }}\end{array}$} & Yes & 67 & 25.9 & 7 & 5.7 & \multirow[t]{2}{*}{$<0.001$} & 47 & 16.5 & 31 & 12.6 & \multirow[t]{2}{*}{0.201} \\
\hline & No & 192 & 74.1 & 115 & 94.3 & & 237 & 83.5 & 215 & 87.4 & \\
\hline \multirow[t]{2}{*}{ NSAID use due to LBP symptoms } & Yes & 99 & 38.2 & 25 & 20.5 & \multirow[t]{2}{*}{$<0.002$} & 111 & 39.1 & 112 & 45.5 & \multirow[t]{2}{*}{0.134} \\
\hline & No & 160 & 61.8 & 97 & 79.5 & & 173 & 60.9 & 134 & 54.5 & \\
\hline \multirow[t]{2}{*}{ Sleeping difficulties due to LBP } & Yes & 13 & 5.0 & 7 & 5.7 & \multirow[t]{2}{*}{0.769} & 33 & 11.6 & 44 & 17.9 & \multirow[t]{2}{*}{$<0.05$} \\
\hline & No & 246 & 95.0 & 115 & 94.3 & & 251 & 88.4 & 202 & 82.1 & \\
\hline \multirow[t]{2}{*}{ Radiating LBP ${ }^{b}$} & Yes & 57 & 22.0 & 19 & 15.6 & \multirow[t]{2}{*}{0.143} & 66 & 23.2 & 37 & 15.0 & \multirow[t]{2}{*}{$<0.02$} \\
\hline & No & 202 & 78.0 & 103 & 84.4 & & 218 & 76.8 & 209 & 85.0 & \\
\hline \multirow[t]{2}{*}{ Operation due to your LBP } & Yes & 2 & 0.8 & 0 & 0.0 & \multirow[t]{2}{*}{0.330} & 1 & 0.4 & 1 & 0.4 & \multirow[t]{2}{*}{0.919} \\
\hline & No & 257 & 99.2 & 122 & 100.0 & & 283 & 99.6 & 245 & 99.6 & \\
\hline \multirow[t]{4}{*}{ LBP origin } & & & & & & \multirow[t]{4}{*}{0.413} & & & & & \multirow[t]{4}{*}{0.653} \\
\hline & Acute $^{c}$ & 46 & 17.8 & 17 & 13.9 & & 23 & 8.1 & 22 & 8.9 & \\
\hline & Overuse $^{d}$ & 185 & 71.4 & 95 & 77.9 & & 239 & 84.2 & 200 & 81.3 & \\
\hline & Both & 28 & 10.8 & 10 & 8.2 & & 22 & 7.7 & 24 & 9.8 & \\
\hline
\end{tabular}

Statistically significant results are indicated in bold

" $p$-value for difference between members and non-members of sports clubs

${ }^{a}$ From a physician, physiotherapist, or chiropractor

bBP that radiates to the lower extremities (buttocks, thigh, knee, lower leg, or foot)

c After injury to low back

d Slowly without injury 
Table 4 Prevalence of NSP in members and non-members of sports clubs

\begin{tabular}{|c|c|c|c|c|c|c|c|c|c|c|c|}
\hline \multirow{3}{*}{ Variables } & \multirow{3}{*}{ Category } & \multicolumn{5}{|c|}{ Boys $(n=772)$} & \multicolumn{5}{|c|}{ Girls $(n=865)$} \\
\hline & & \multicolumn{2}{|c|}{ Member } & \multicolumn{2}{|c|}{ Non-member } & \multirow[t]{2}{*}{$P$-value ${ }^{*}$} & \multicolumn{2}{|c|}{ Member } & \multicolumn{2}{|c|}{ Non-member } & \multirow[t]{2}{*}{$P$-value } \\
\hline & & $n$ & $\%$ & $n$ & $\%$ & & $n$ & $\%$ & $n$ & $\%$ & \\
\hline \multirow[t]{2}{*}{ NSP $P^{a}$} & Yes & 130 & 26.5 & 81 & 28.8 & \multirow[t]{2}{*}{0.481} & 222 & 47.1 & 236 & 59.9 & \multirow[t]{2}{*}{$<0.001$} \\
\hline & No & 361 & 73.5 & 200 & 71.2 & & 249 & 52.9 & 158 & 40.1 & \\
\hline \multirow[t]{2}{*}{ Frequent NSP $\mathrm{P}^{\mathrm{b}}$} & Yes & 19 & 3.9 & 23 & 8.2 & \multirow[t]{2}{*}{$<0.02$} & 67 & 14.2 & 104 & 26.4 & \multirow[t]{2}{*}{$<0.001$} \\
\hline & No & 472 & 96.1 & 258 & 91.8 & & 404 & 85.8 & 290 & 73.6 & \\
\hline
\end{tabular}

Statistically significant results are indicated in bold

${ }^{*} p$-value for difference between members and non-members of sports clubs

${ }^{a}$ NSP more than once a month

${ }^{b}$ NSP at least once a week

days during the training season decreased the odds of having LBP in boys.

\section{Risk factors for neck and shoulder pain}

Adjusted odds ratios of health (Table 8), health behaviour (Table 9), and training characteristics (Table 10) are shown in the tables. The odds for self-reported NSP were increased by having chronic disease(s) (OR 1.85, 95 \% CI 1.23-2.80 for boys and OR 1.49, 95 \% CI 1.052.10 for girls), and also with reporting low back, thoracic spine, and upper limb pain (Table 8).

Higher screen time, as calculated per additional hour of screen time (computer games, TV/DVD, phone, Internet) during leisure time, slightly increased the odds of NSP in boys, as presented in Table 9 (OR 1.05, 95 \% CI 1.00-1.10). For girls, the increased odds were not statistically significant (also shown in Table 9). However, analysis also detected a significant increase in the odds for NSP among girls when screen time exceeded $4 \mathrm{~h}$ /day (OR 1.39, $95 \% \mathrm{CI}$ 1.05-1.85). Smoking increased the odds of NSP (OR 1.65, $95 \%$ CI 1.04-2.59, Table 9) in girls. Sports club membership was associated with a lower risk for frequent NSP in girls (OR 0.52, 95 \% CI 0.33-0.82). Associations were not found between NSP and training characteristics (Table 10) other than an additional year of active playing/practicing slightly increased the odds of NSP in girls (OR 1.07, $95 \%$ CI 1.00-1.14).

\section{Discussion}

In this multidisciplinary multicenter study, we investigated the prevalence of self-reported low back pain and neck and shoulder pain, and the related factors in members and non-members of adolescents' sports clubs. Our findings show that self-reported low back pain (LBP) and neck and shoulder pain (NSP) are already common among adolescents. Girls seem to be at a higher risk for

Table 5 Associations between LBP and health variables in 14 to 16 year old Finnish adolescents

\begin{tabular}{|c|c|c|c|c|c|c|c|c|c|}
\hline \multirow{3}{*}{ Variables } & \multirow{3}{*}{ Category } & \multicolumn{4}{|l|}{$\mathrm{LBP}^{\mathrm{a}}$} & \multicolumn{4}{|c|}{ Frequent $\mathrm{LBP}^{b}$} \\
\hline & & \multicolumn{2}{|c|}{ Boys $(n=768)$} & \multicolumn{2}{|c|}{ Girls $(n=856)$} & \multicolumn{2}{|c|}{ Boys $(n=768)$} & \multicolumn{2}{|c|}{ Girls $(n=856)$} \\
\hline & & $\mathrm{OR}^{\mathrm{c}}$ & $95 \% \mathrm{Cl}$ & $\mathrm{OR}^{\mathrm{C}}$ & $95 \% \mathrm{Cl}$ & $\mathrm{OR}^{\mathrm{C}}$ & $95 \% \mathrm{Cl}$ & $\mathrm{OR}^{c}$ & $95 \% \mathrm{Cl}$ \\
\hline \multirow[t]{2}{*}{ Chronic diseases $^{d}$} & No & \multicolumn{2}{|c|}{1 (referent) } & \multicolumn{2}{|c|}{1 (referent) } & \multicolumn{2}{|c|}{1 (referent) } & \multicolumn{2}{|c|}{1 (referent) } \\
\hline & Yes & 0.71 & $(0.44-1.13)$ & 1.36 & $(0.95-1.96)$ & 0.72 & $(0.34-1.55)$ & 1.38 & $(0.84-2.25)$ \\
\hline BMI & & 1.04 & $(0.97-1.11)$ & 0.96 & $(0.91-1.02)$ & 1.14 & $(0.44-2.99)$ & 1.01 & $(0.94-1.09)$ \\
\hline \multirow[t]{2}{*}{ Neck pain } & No & \multicolumn{2}{|c|}{1 (referent) } & \multicolumn{2}{|c|}{1 (referent) } & \multicolumn{2}{|c|}{1 (referent) } & \multicolumn{2}{|c|}{1 (referent) } \\
\hline & $Y_{e s}^{e}$ & 1.83 & $(1.13-2.96)$ & 2.13 & $(1.47-3.09)$ & 1.65 & $(0.72-3.77)$ & 1.74 & $(0.91-3.33)$ \\
\hline \multirow[t]{2}{*}{ Thoracic spine pain } & No & \multicolumn{2}{|c|}{1 (referent) } & \multicolumn{2}{|c|}{1 (referent) } & \multicolumn{2}{|c|}{1 (referent) } & \multicolumn{2}{|c|}{1 (referent) } \\
\hline & Yes $^{\mathrm{e}}$ & 9.39 & $(5.39-16.34)$ & 6.31 & $(4.15-9.59)$ & 2.88 & $(1.22-6.82)$ & 4.49 & $(2.61-7.74)$ \\
\hline \multirow[t]{2}{*}{ Upper limb pain } & No & \multicolumn{2}{|c|}{1 (referent) } & \multicolumn{2}{|c|}{1 (referent) } & \multicolumn{2}{|c|}{1 (referent) } & \multicolumn{2}{|c|}{1 (referent) } \\
\hline & $Y_{e s}^{e}$ & 1.87 & $(1.02-3.44)$ & 1.41 & $(0.90-2.12)$ & 0.77 & $(0.26-2.27)$ & 1.95 & $(1.12-3.40)$ \\
\hline \multirow[t]{2}{*}{ Lower limb pain } & No & \multicolumn{2}{|c|}{1 (referent) } & \multicolumn{2}{|c|}{1 (referent) } & \multicolumn{2}{|c|}{1 (referent) } & \multicolumn{2}{|c|}{1 (referent) } \\
\hline & $Y_{e s}^{e}$ & 1.74 & $(1.02-2.96)$ & 1.53 & $(1.03-2.27)$ & 1.52 & $(0.61-3.76)$ & 1.42 & $(0.82-2.46)$ \\
\hline
\end{tabular}

Statistically significant results are indicated in bold

aBP more than once a month during the last 3 months

${ }^{b}$ LBP at least once a week during the last 3 months

'Binary logistic regression was used and all variables were included in the same model. Analyses were adjusted by age, BMI, chronic diseases, smoking, school attainment level

${ }^{\mathrm{d}}$ Allergy, asthma, diabetes, epilepsy, heart condition, etc.

${ }^{\mathrm{e}}$ At least once a month 
Table 6 Associations between LBP and health behaviour variables in 14 to 16 year old Finnish adolescents

\begin{tabular}{|c|c|c|c|c|c|c|c|c|c|}
\hline \multirow{3}{*}{ Variables } & \multirow{3}{*}{ Category } & \multicolumn{4}{|l|}{$\mathrm{LBP}^{\mathrm{a}}$} & \multicolumn{4}{|c|}{ Frequent $\mathrm{LBP}^{\mathrm{b}}$} \\
\hline & & \multicolumn{2}{|c|}{ Boys $(n=768)$} & \multicolumn{2}{|c|}{ Girls $(n=856)$} & \multicolumn{2}{|c|}{ Boys $(n=768)$} & \multicolumn{2}{|c|}{ Girls $(n=856)$} \\
\hline & & $\mathrm{OR}^{c}$ & $95 \% \mathrm{Cl}$ & $\overline{\mathrm{OR}^{c}}$ & $95 \% \mathrm{Cl}$ & $\mathrm{OR}^{c}$ & $95 \% \mathrm{Cl}$ & $\mathrm{OR}^{\mathrm{C}}$ & $95 \% \mathrm{Cl}$ \\
\hline Screen time $^{d}$ & & 1.07 & $(1.01-1.12)$ & 1.06 & $(1.01-1.10)$ & 1.04 & $(0.99-1.10)$ & 1.03 & $(0.99-1.09)$ \\
\hline \multirow[t]{3}{*}{ Leisure time $\mathrm{PA}^{\mathrm{e}}$} & Approx. $<30 \mathrm{~min} /$ week & \multicolumn{2}{|c|}{1 (referent) } & \multicolumn{2}{|c|}{1 (referent) } & \multicolumn{2}{|c|}{1 (referent) } & \multicolumn{2}{|c|}{1 (referent) } \\
\hline & Approx. 1-3 h/week & 1.38 & $(0.58-3.29)$ & 1.31 & $(0.74-2.31)$ & 2.04 & $(0.40-10.27)$ & 0.79 & $(0.35-1.79)$ \\
\hline & Approx.4-6 h/week or more & 1.56 & $(0.63-3.82)$ & 1.75 & $(0.95-3.23)$ & 1.11 & $(0.20-6.10)$ & 1.20 & $(0.50-2.85)$ \\
\hline \multirow[t]{2}{*}{ Sports club membership } & No & \multicolumn{2}{|c|}{1 (referent) } & \multicolumn{2}{|c|}{1 (referent) } & \multicolumn{2}{|c|}{1 (referent) } & \multicolumn{2}{|c|}{1 (referent) } \\
\hline & Yes & 2.35 & $(1.48-3.72)$ & 0.97 & $(0.67-1.42)$ & 2.73 & $(1.17-6.34)$ & 0.99 & $(0.56-1.76)$ \\
\hline \multirow[t]{3}{*}{ Use of alcohol } & $<1 \times$ month & \multicolumn{2}{|c|}{1 (referent) } & \multicolumn{2}{|c|}{1 (referent) } & \multicolumn{2}{|c|}{1 (referent) } & \multicolumn{2}{|c|}{1 (referent) } \\
\hline & $1 \times$ month & 1.15 & $(0.59-2.22)$ & 1.40 & $(0.77-2.55)$ & 1.39 & $(0.45-4.29)$ & 1.63 & $(0.76-3.50)$ \\
\hline & $\geq 2-3 \times$ month & 2.25 & $(1.04-4.90)$ & 1.17 & $(0.61-2.25)$ & 2.73 & $(0.87-8.60)$ & 0.57 & $(0.19-1.75)$ \\
\hline \multirow[t]{2}{*}{ Smoking } & No & \multicolumn{2}{|c|}{1 (referent) } & \multicolumn{2}{|c|}{1 (referent) } & \multicolumn{2}{|c|}{1 (referent) } & \multicolumn{2}{|c|}{1 (referent) } \\
\hline & Yes & 2.32 & $(1.29-4.19)$ & 1.96 & $(1.26-3.04)$ & 1.42 & $(0.53-3.78)$ & 1.46 & $(0.78-2.76)$ \\
\hline
\end{tabular}

Statistically significant results are indicated in bold

${ }^{\mathrm{a}} \mathrm{LBP}$ more than once a month during the last 3 months

${ }^{\mathrm{b}} \mathrm{LBP}$ at least once a week during the last 3 months

'Binary logistic regression was used and all variables were included in the same model. Analyses were adjusted by age, BMI, chronic diseases, smoking, school attainment level

${ }^{\mathrm{d}} \mathrm{TV}$, computer, computer/console games, phone, tablet use, OR calculated per additional hour of screen time

e Intensity: breathlessness and sweating

reporting LBP and NSP. Our results also suggest that the prevalence of LBP is higher in boys who participate in organized sports club activities. On the other hand, sports club members seem to suffer NSP less frequently than non-members do in general.

The strength of this study was the representative sample of adolescents, who were aged 14-16 years and from different regions and sizes of municipality. The sports club sample comprised the ten most popular sports in Finland. Organized sports clubs are the main setting for leisure-time physical activity in adolescents, especially in the Nordic countries. In Finland, nearly half (46 \%) of children and adolescents aged 10-16 years take part in organized sports club activities [22]. Due to their wide reach and the informal educational nature, sports clubs offer a potential setting for health promotion [23]. However, even though sports clubs are positively oriented towards the idea of health promotion, the clubs' practices have been shown to be limited and directed mainly towards sports performance and less towards other areas of health [24].

It is a common belief that those who participate in sports club activities automatically have a more physically active and healthy lifestyle than non-members. Research findings on these issues are, however, inconsistent. Three quarters of the general population of Finnish children and adolescents aged 11-15 [22, 25] and one third of sports club members in the Nordic countries do not meet the recommended level of physical activity [26-28]. In the present study, sports club members were significantly more active than non-members; nevertheless, $16 \%$ of the members reported only approximately $1-3 \mathrm{~h}$ of leisure time activity per week.

We found a $49.5 \%$ lifetime prevalence of self-reported LBP in boys, and the same prevalence for girls was $61.3 \%$,

Table 7 Associations between LBP and training characteristics in 14 to 16 year old sports club members

\begin{tabular}{|c|c|c|c|c|}
\hline \multirow[b]{2}{*}{ Training Characteristics } & \multicolumn{2}{|l|}{ Boys } & \multicolumn{2}{|l|}{ Girls } \\
\hline & $\overline{O R^{a}}$ & $95 \% \mathrm{Cl}$ & $\mathrm{OR}^{a}$ & $95 \% \mathrm{Cl}$ \\
\hline Active playing/practicing years (boys $n=488$, girls $n=465$ ) & 1.05 & $(0.97-1.13)$ & 1.07 & $(1.00-1.14)$ \\
\hline Training hours per week during training season (boys $n=486$, girls $n=463$ ) & 1.05 & $(1.01-1.09)$ & 1.01 & $(0.96-1.05)$ \\
\hline Training hours per week during competition season (boys $n=482$, girls $n=448$ ) & 1.03 & $(0.99-1.08)$ & 1.03 & $(0.99-1.08)$ \\
\hline Number of competitions/games during previous 12 months (boys $n=485$, girls $n=462$ ) & 1.01 & $(1.00-1.02)$ & 1.00 & $(0.98-1.01)$ \\
\hline Number of rest days during training season (boys $n=483$, girls $n=461$ ) & 0.78 & $(0.65-0.94)$ & 0.96 & $(0.83-1.11)$ \\
\hline Number of rest days during competition season (boys $n=480$, girls $n=459$ ) & 0.79 & $(0.66-0.94)$ & 0.84 & $(0.72-1.00)$ \\
\hline
\end{tabular}

Statistically significant results are indicated in bold

${ }^{a}$ All training variables analysed in separate models. Adjusted by age, BMl, chronic diseases, smoking, school attainment level 
Table 8 Associations between NSP and health variables in 14 to 16 year old Finnish adolescents

\begin{tabular}{|c|c|c|c|c|c|c|c|c|c|}
\hline \multirow{3}{*}{ Variables } & \multirow{3}{*}{ Category } & \multicolumn{4}{|l|}{ NSPa } & \multicolumn{4}{|c|}{ Frequent NSP } \\
\hline & & \multicolumn{2}{|c|}{ Boys $(n=768)$} & \multicolumn{2}{|c|}{ Girls $(n=856)$} & \multicolumn{2}{|c|}{ Boys $(n=768)$} & \multicolumn{2}{|c|}{ Girls $(n=856)$} \\
\hline & & $\overline{\mathrm{OR}^{c}}$ & $95 \% \mathrm{Cl}$ & $\overline{\mathrm{OR}^{c}}$ & $95 \% \mathrm{Cl}$ & $\overline{\mathrm{OR}^{c}}$ & $95 \% \mathrm{Cl}$ & $\overline{\mathrm{OR}^{c}}$ & $95 \% \mathrm{Cl}$ \\
\hline \multirow[t]{2}{*}{ Chronic diseases $^{d}$} & No & \multicolumn{2}{|c|}{1 (referent) } & \multicolumn{2}{|c|}{1 (referent) } & \multicolumn{2}{|c|}{1 (referent) } & \multicolumn{2}{|c|}{1 (referent) } \\
\hline & Yes & 1.85 & $(1.23-2.80)$ & 1.49 & $(1.05-2.10)$ & 1.00 & $(0.46-2.18)$ & 1.21 & $(0.81-1.79)$ \\
\hline BMI & & 1.03 & $(0.97-1.10)$ & 1.03 & $(0.97-1.08)$ & 1.02 & $(0.91-1.15)$ & 1.07 & $(1.01-1.13)$ \\
\hline \multirow[t]{2}{*}{ Low back pain } & No & \multicolumn{2}{|c|}{1 (referent) } & \multicolumn{2}{|c|}{1 (referent) } & \multicolumn{2}{|c|}{1 (referent) } & \multicolumn{2}{|c|}{1 (referent) } \\
\hline & Yes $^{e}$ & 1.88 & $(1.16-3.03)$ & 2.15 & $(1.48-3.11)$ & 1.84 & $(0.73-4.66)$ & 1.88 & $(1.22-2.91)$ \\
\hline \multirow[t]{2}{*}{ Thoracic spine pain } & No & \multicolumn{2}{|c|}{1 (referent) } & \multicolumn{2}{|c|}{1 (referent) } & \multicolumn{2}{|c|}{1 (referent) } & \multicolumn{2}{|c|}{1 (referent) } \\
\hline & $Y_{e s}{ }^{e}$ & 3.53 & $(1.97-6.33)$ & 4.87 & $(2.92-8.11)$ & 4.91 & $(1.94-12.42)$ & 3.87 & $(2.48-6.06)$ \\
\hline \multirow[t]{2}{*}{ Upper limb pain } & No & \multicolumn{2}{|c|}{1 (referent) } & \multicolumn{2}{|c|}{1 (referent) } & \multicolumn{2}{|c|}{1 (referent) } & \multicolumn{2}{|c|}{1 (referent) } \\
\hline & Yes $^{e}$ & 6.47 & $(3.73-11.23)$ & 4.00 & $(2.39-6.61)$ & 1.00 & $(0.35-2.88)$ & 1.94 & $(1.21-3.10)$ \\
\hline \multirow[t]{2}{*}{ Lower limb pain } & No & \multicolumn{2}{|c|}{1 (referent) } & \multicolumn{2}{|c|}{1 (referent) } & \multicolumn{2}{|c|}{1 (referent) } & \multicolumn{2}{|c|}{1 (referent) } \\
\hline & $\mathrm{Yes}^{\mathrm{e}}$ & 1.39 & $(0.83-2.33)$ & 1.44 & $(0.98-2.12)$ & 0.65 & $(0.23-1.88)$ & 1.07 & $(0.68-1.68)$ \\
\hline
\end{tabular}

Statistically significant results are indicated in bold

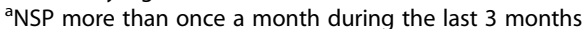

${ }^{\mathrm{b}} \mathrm{NSP}$ at least once a week during the last 3 months

'Binary logistic regression was used and all variables were included in the same model. Analyses were adjusted by age, BMI, chronic diseases, smoking, school attainment level

${ }^{\mathrm{d} A l l e r g y, ~ a s t h m a, ~ d i a b e t e s, ~ e p i l e p s y, ~ h e a r t ~ c o n d i t i o n, ~ e t c . ~}$

${ }^{\mathrm{e}}$ At least once a month

which is in line with the findings of Harreby at al. [29], who investigated the risk factors of LBP in a cohort of 1389 Danish children aged $12-16$ years ( $49.8 \%$ in boys and $67.4 \%$ in girls) and had similar definition of LBP as our study. Van Gent et al. [30] reported 3-month prevalence of LBP in adolescents aged $12-14$ years $(n=745)$ as $53.8 \%$ for girls and $39.4 \%$ for boys. In the present study, the prevalence of frequent LBP was in line with the results of severe LBP in the study Van Gent et al. [30] (11.0\% vs $9.5 \%$ in girls and $5.4 \%$ vs $4.5 \%$ in boys, respectively). Van Gent et al. [30] defined LBP complaints severe if they bothered the children daily, demanded medication use or affected normal functioning, which is somewhat different than in our study. In our study LBP was defined as"ache or pain in the low back" and frequent LBP was reported to occur at least once a week.

Table 9 Associations between NSP and health behaviour variables in 14 to 16 year old Finnish adolescents

\begin{tabular}{|c|c|c|c|c|c|c|c|c|c|}
\hline \multirow{3}{*}{ Variables } & \multirow{3}{*}{ Category } & \multicolumn{4}{|l|}{ NSPa } & \multicolumn{4}{|c|}{ Frequent NSP ${ }^{b}$} \\
\hline & & \multicolumn{2}{|c|}{ Boys $(n=768)$} & \multicolumn{2}{|c|}{ Girls $(n=856)$} & \multicolumn{2}{|c|}{ Boys $(n=768)$} & \multicolumn{2}{|c|}{ Girls $(n=856)$} \\
\hline & & $\overline{\mathrm{OR}^{c}}$ & $95 \% \mathrm{Cl}$ & $\overline{\mathrm{OR}^{\mathrm{c}}}$ & $95 \% \mathrm{Cl}$ & $\overline{\mathrm{OR}^{c}}$ & $95 \% \mathrm{Cl}$ & $\overline{\mathrm{OR}^{\mathrm{c}}}$ & $95 \% \mathrm{Cl}$ \\
\hline Screen time ${ }^{d}$ & & 1.05 & $(1.00-1.10)$ & 1.03 & $(0.98-1.07)$ & 1.03 & $(0.98-1.09)$ & 1.02 & $(0.98-1.06)$ \\
\hline \multirow[t]{3}{*}{ Leisure time $\mathrm{PA}^{\mathrm{e}}$} & Approx. $<30 \mathrm{~min} /$ week & \multicolumn{2}{|c|}{1 (referent) } & \multicolumn{2}{|c|}{1 (referent) } & \multicolumn{2}{|c|}{1 (referent) } & \multicolumn{2}{|c|}{1 (referent) } \\
\hline & Approx. 1-3 h/week & 0.81 & $(0.40-1.65)$ & 1.37 & $(0.80-2.33)$ & 1.94 & $(0.53-7.11)$ & 1.29 & $(0.69-2.39)$ \\
\hline & Approx. 4-6 h/week or more & 0.79 & $(0.37-1.66)$ & 1.07 & $(0.60-1.90)$ & 1.27 & $(0.31-5.15)$ & 1.23 & $(0.62-2.41)$ \\
\hline \multirow[t]{2}{*}{ Sports club membership } & No & \multicolumn{2}{|c|}{1 (referent) } & \multicolumn{2}{|c|}{1 (referent) } & \multicolumn{2}{|c|}{1 (referent) } & \multicolumn{2}{|c|}{1 (referent) } \\
\hline & Yes & 1.09 & $(0.72-1.65)$ & 0.76 & $(0.53-1.10)$ & 0.64 & $(0.30-1.37)$ & 0.52 & $(0.33-0.82)$ \\
\hline \multirow[t]{3}{*}{ Use of alcohol } & $<1 \times$ month & \multicolumn{2}{|c|}{1 (referent) } & \multicolumn{2}{|c|}{1 (referent) } & \multicolumn{2}{|c|}{1 (referent) } & \multicolumn{2}{|c|}{1 (referent) } \\
\hline & $1 \times$ month & 1.70 & $(0.91-3.17)$ & 1.84 & $(0.97-3.49)$ & 1.46 & $(0.47-4.54)$ & 1.34 & $(0.68-2.62)$ \\
\hline & $\geq 2-3 \times$ month & 2.75 & $(1.29-5.83)$ & 1.52 & $(0.77-2.99)$ & 2.73 & $(0.92-8.09)$ & 0.96 & $(0.45-2.06)$ \\
\hline \multirow[t]{2}{*}{ Smoking } & No & \multicolumn{2}{|c|}{1 (referent) } & \multicolumn{2}{|c|}{1 (referent) } & \multicolumn{2}{|c|}{1 (referent) } & \multicolumn{2}{|c|}{1 (referent) } \\
\hline & Yes & 1.36 & $(0.76-2.43)$ & 1.65 & $(1.04-2.59)$ & 1.56 & $(0.61-3.98)$ & 1.42 & $(0.87-2.32)$ \\
\hline
\end{tabular}

Statistically significant results are indicated in bold

${ }^{a}$ NSP more than once a month during the last 3 months

${ }^{\mathrm{b}} \mathrm{NSP}$ at least once a week during the last 3 months

${ }^{\mathrm{C} B i n a r y}$ logistic regression was used and all variables were included in the same model. Analyses were adjusted by age, BMI, chronic diseases, smoking, school attainment level

${ }^{\mathrm{d}} \mathrm{TV}$, computer, computer/console games, phone, tablet use, OR calculated per additional hour of screen time

entensity: breathlessness and sweating 
Table 10 Associations between NSP and training characteristics in 14 to 16 year old sports club members

\begin{tabular}{|c|c|c|c|c|}
\hline \multirow[b]{2}{*}{ Training Characteristics } & \multicolumn{2}{|c|}{ Boys } & \multicolumn{2}{|l|}{ Girls } \\
\hline & $\overline{O R^{a}}$ & $95 \% \mathrm{Cl}$ & $\overline{\mathrm{OR}^{\mathrm{a}}}$ & $95 \% \mathrm{Cl}$ \\
\hline Active playing/practicing years (boys $n=488$, girls $n=465$ ) & 0.94 & $(0.87-1.01)$ & 1.07 & $(1.00-1.14)$ \\
\hline Training hours per week during training season (boys $n=486$, girls $n=463$ ) & 1.00 & $(0.96-1.04)$ & 0.98 & $(0.94-1.02)$ \\
\hline Training hours per week during competition season (boys $n=482$, girls $n=448$ ) & 0.98 & $(0.93-1.02)$ & 1.00 & $(0.96-1.04)$ \\
\hline Number of competitions/games during previous 12 months (boys $n=485$, girls $n=462$ ) & 1.00 & $(0.99-1.00)$ & 1.00 & $(0.99-1.01)$ \\
\hline Number of rest days during training season (boys $n=483$, girls $n=461$ ) & 1.10 & $(0.92-1.30)$ & 1.05 & $(0.91-1.21)$ \\
\hline Number of rest days during competition season (boys $n=480$, girls $n=459$ ) & 1.15 & $(0.96-1.37)$ & 0.96 & $(0.83-1.10)$ \\
\hline
\end{tabular}

Statistically significant results are indicated in bold

${ }^{a}$ All training variables analysed in separate models. Adjusted by age, BMI, chronic diseases, smoking, school attainment level

Van Gent et al. [30] reported that among 12-14-yearsold, the prevalence of severe NSP is $6.5 \%$ for girls and $5.0 \%$ for boys. Diepenmaat et al. [13] reported that among $12-16$-year-olds, the prevalence of frequent NSP (more than 4 days a month) is $14.2 \%$ for girls and $8.7 \%$ for boys. Myrtveit et al. [31] reported that among 18-year-olds, weekly NSP was suffered by $28 \%$ of girls and $11 \%$ of boys. Similarly, Ståhl et al. [4] found a $19 \%$ prevalence of weekly neck pain among of 13-16-year-old boys and girls. Our findings on the prevalence of frequent NSP are in line with these previous findings. However, the prevalence of NSP in girls was higher in our sample compared to previous studies $[13,30]$.

It has been suggested that the relationship between LBP and physical activity is U-shaped [32]. Some studies have found that as the intensity or amount of physical activity increases, so does the risk of LBP in the adolescents $[7,10,12,33]$. Some studies have not been able to find an association between physical activity and LBP $[13,14,30,34]$ or the development of neck and upper limb/shoulder pain [13, 35-38]. In a recent prospective population-based cohort study among 19-21-year-old men, moderate physical activity and a good fitness level were found to protect the subjects from LBP [39]. Physical activity has been reported to be associated with a reduced risk for NSP [31]. Wedderkopp and et al. [36] did not notice significant increases in the odds of neck pain when they compared physical activity (low, mid, high) measured objectively with an accelerometer in 9-year-old children. However, they noticed that 9-year-old children with the lowest levels of physical activity were four times more likely to have low back pain 3 years later than the children with the highest levels of physical activity [36].

Mogensen et al. [34] investigated the difference of the 1 -month prevalence of low back pain and neck pain in adolescents (12-13-years-old) participating in sports and those who did not take part in any sport. They found no difference between the groups for LBP ( $40 \%$ vs $39 \%$ ) or neck pain (13\% vs $11 \%)$. Even though we did not find statistically significant associations between self-reported leisure-time physical activity and LBP or NSP in the present study, we did find a significantly higher prevalence of LBP in male sports club members and a higher prevalence of NSP in non-members in general. According to a prospective study, athletes participating in sports club activities at least twice a week reported significantly more LBP than non-athletes ( $n=116$, age range 10.3-13.3) [40]. The higher prevalence of LBP in boys who are sports club members might be due to the higher volume and intensity of exercise. The increased prevalence of LBP in male sports club members might be due to insufficient recovery, as suggested by our finding on the association between LBP and the number of rest days.

In the present study, the majority of the subjects both members and non-members - reported the origin of LBP to be overuse, and the results are in line with previous reports within athletic and general populations $[11,40-43]$. In addition, previous results $[4,44]$ on concomitant pain being more common than single LBP or single NSP are supported by our findings.

With regard to gender, our results are in line with previous results. In general, girls are at a higher risk for developing LBP $[2,3,9,29]$ and NSP [2-4, 9, 30, 31]. However, the recent meta-analysis of LBP in children and adolescents by Calvo-Muñoz et al. [5] and the study by Schmidt et al. [45] - who studied the prevalence of LBP in adolescent athletes - found no association between gender and LBP. We found that the girls' odds of having frequent LBP and frequent NSP were 2.33 - (95\% CI 1.58-3.45) and 4.45-times (95 \% CI 3.08-6.40) higher than the boys' odds.

Interestingly our results showed a trend towards selfreported LBP being more common in non-members in girls, contrary what was seen among the boys. This might simply be a consequence of boys participating more frequently in sports with higher spinal loads (flexion and rotation), such as ice hockey and football. We found higher prevalence of frequent NSP in non-members. In relation to previous studies that have found frequent computerrelated activities to increase the risk of NSP and LBP in 
adolescents [2] it could be speculated that the increased prevalence in the present study may be at least partly associated with the higher screen time reported by the nonmembers. On the other hand, NSP has also been associated with depressive symptoms and stress in a study where computer use was not found to be significantly associated with NSP or LBP [13].

It could be expected that when the amount of rest and recovery time decreases, the incidence in overuse injuries in particular increases. High frequency of training and lack of rest days are possible risk factors that sports clubs can control and thus modify the predisposing factors for injuries. In this study, no significant associations between training exposure hours per week and LBP were found in girls, which is in accordance with findings of the study by Tunas et al. [11]. However, we found a negative association between LBP and rest days and a positive association between LBP and number of competitions, and training hours in males. The number of rest days was also associated with LBP in girls, the association being negative. Schmidt et al. [45] found a statistically significant trend towards an increased prevalence of LBP in those athletes who were training the most. Ristolainen et al. [46] found that elite athletes (aged 15-35) with less than two rest days per week during the training season were more than five times more likely to report an overuse injury (95\% CI 1.89-14.06, $P=0.001$ ). It is therefore important at the sports club-level to tackle the challenge of how to minimize the possibility of overload and to decrease the incidence of overuse injuries.

There are some limitations in the present study that must be acknowledged. Due to the cross-sectional design of the study, one must be cautious in drawing conclusions, especially concerning causality -that is, to differentiate the associated factors as predisposing factors or simply consequences. For example, pain may have affected training frequency or duration and influenced the physical activity or inactivity of the study subjects. In addition, there is a possible recall bias as with retrospective designs, the ability of the subject to remember and report the information correctly is a potential issue. The validity of the surveys was not studied; however, the questionnaires used in these surveys were compiled from previously validated questions in other similar studies of school-aged adolescents [16-20].

Also psychosocial factors have been shown to be associated with LBP and NSP in adolescents [44, 47]. The lacking of these variables as potential confounders could have influenced the results of this study as screen time has been shown to be associated with symptoms of depression and anxiety in adolescent [48].

\section{Conclusions}

Self-reported low back pain and neck and shoulder pain are common among 14-16-year-olds. The prevalence of LBP was higher in male sports club members and the prevalence of NSP was higher among non-members in general. It also seems that higher screen time is weakly associated with musculoskeletal symptoms of the back, neck, and shoulder regions among adolescents.

\section{Additional file}

Additional file 1: HPSC Health behaviour survey. (PDF 197 kb)

Additional file 2: HPSC MSK survey. (PDF 254 kb)

Abbreviations

LBP, low back pain; NSP, neck and shoulder pain

Acknowledgements

Not applicable.

Funding

This study was financially supported by the Finnish Ministry of Education and Culture. (grant number: 6/091/2011).

Availability of data and materials

The data can not be shared because permission was not asked from the participants or their parents.

Authors' contributions

All authors contributed to study conception and design. SK and JP coordinated and managed all parts of the study. MR carried out the literature search. SK and JV conducted data collection and performed preliminary data preparations. MR and JV conducted data analyses and all the authors contributed to the interpretation of data. MR and JP wrote the first draft of the paper and all authors provided substantive feedback on the paper and contributed to the final manuscript. All authors have approved the submitted version of the manuscript. JP is the guarantor.

\section{Authors' information}

Not applicable.

\section{Competing interests}

All authors have completed the Unified Competing Interest form at www.icmje.org/coi_disclosure.pdf (available on request from the corresponding author) and declare that all authors had: (1) no financial support for the submitted work from anyone other than their employer; (2) no financial relationships with commercial entities that might have an interest in the submitted work; (3) no spouses, partners, or children with relationships with commercial entities that might have an interest in the submitted work; and (4) no non-financial interests that may be relevant to the submitted work.

\section{Consent for publication}

Not applicable.

\section{Ethics approval and consent to participate}

Ethical approval was received from the Ethics Committee of Health Care District of Central Finland (record number 23U/2012). All sports clubs participated in the study by free will and were notified that they had a right to refuse to participate and withdraw from the study at any time. A written consent was obtained as required by the ethical statement.

\section{Author details}

${ }^{1}$ Tampere Research Center of Sports Medicine, P.O. Box 30FI-33501 Tampere, Finland. ${ }^{2}$ Clinic for Sports and Exercise Medicine, Alppikatu 2, Fl-00530 Helsinki, Finland. ${ }^{3}$ Paavo Nurmi Centre \& Department of Health and Physical Activity, University of Turku, Kiinamyllynkatu 10, Fl-20520 Turku, Finland. ${ }^{4}$ Department of Sports and Exercise Clinic, Oulu Deaconess Institute, Albertinkatu 18, Fl-90100 Oulu, Finland. ${ }^{5}$ Department of Health Sciences, University of Jyväskylä, P.O. Box 35Fl-40014 Jyväskylä, Finland. ${ }^{6}$ Kuopio Research Institute of Exercise Medicine, Haapaniementie 16, Fl-70100 Kuopio, Finland. ${ }^{7}$ LIKES Foundation for Sports and Health Sciences and Mehiläinen 
Physical Activity Clinic, P.O. Box 35Fl-40720 Jyväskylä, Finland. ${ }^{8}$ UKK Institute for Health Promotion Research, P.O. Box 30FI-33501 Tampere, Finland. ${ }^{9}$ University of Oulu, Center for Life Course Health Research, Oulu, Finland. ${ }^{10}$ Medical Research Center, University of Oulu and University Hospital of Oulu, Oulu, Finland.

Received: 7 January 2016 Accepted: 2 June 2016

Published online: 01 July 2016

\section{References}

1. Statistical yearbook of the social insurance institution 2013. Kansaneläkelaitos The Social Insurance Institution of Finland. 2015. http://www.kela.fi/documents/ 10180/1630875/Statistical_Yearbook_of_the_Social_Insurance_Institution_2013. pdf/0578574a-2b93-4ca5-acof-650f12d15141. Accessed 2 Aug 2015.

2. Hakala P, Rimpelä A, Salminen JJ, Virtanen SM, Rimpelä M. Back, neck, and shoulder pain in Finnish adolescents: national cross sectional surveys. Brit Med J. 2002;325:743-5.

3. Hakala PT, Rimpelä AH, Saarni LA, Salminen JJ. Frequent computer-related activities increase the risk of neck-shoulder and low back pain in adolescents. Eur J Public Health. 2006;16(5):536-41.

4. Ståhl M, Mlkkelson M, Kautianen H, Häkkinen A, Ylinen J, Salminen JJ. Neck pain in adolescence. A 4-year follow-up of pain-free preadolescents. Pain. 2004;110:427-31

5. Calvo-Muñoz I, Gomez-Conesa A, Sanchez-Meca J. Prevalence of low back pain in children and adolescents: A meta-analysis. BMC Pediatr. 2013;13:14.

6. Taimela S, Kujala UM, Salminen JJ, Viljanen T. The prevalence of low back pain among children and adolescents. A nationwide, cohort-based questionnaire survey in Finland. Spine. 1997;22:1132-6.

7. Auvinen J, Tammelin T, Taimela S, Zitting P, Karppinen J. Associations of physical activity and inactivity with low back pain in adolescents. Scand J Med Sci Sports. 2008;18(2):188-94.

8. Hestbaek LDC, Leboeuf-Yde CDC, Kyvik KO, Manniche CDMS. The course of low back pain from adolescence to adulthood: Eight-year follow-up of 9600 twins. Spine. 2006;31(4):468-72.

9. Ståhl M, El-Metwally A, Rimpelä A. Time trends in single versus concomitant neck and back pain in finnish adolescents: results from national cross-sectional surveys from 1991 to 2011. BMC Musculoskelet Disord. 2014;15:296.

10. Hoskins W, Pollard H, Daff C, et al. Low back pain in junior Australian rules football: A cross-sectional survey of elite juniors, non- elite juniors and nonfootball playing controls. BMC Musculoskelet Disord. 2010;11:241.

11. Tunas P, Nilstad A, Myklebust G. Low back pain in female elite football and handball players compared with an active control group. Knee Surg Sports Traumatol Arthrosc. 2014 May 18. [Epub ahead of print]

12. Sato $T$, Ito $T$, Hirano $T$, et al. Low back pain in childhood and adolescence: Assessment of sports activities. Eur Spine J. 2011;20(1):94-9.

13. Diepenmaat ACM, van der Wal MF, de Vet HCW, Hirasing RA. Neck/shoulder, low back, and arm pain in relation to computer use, physical activity, stress, and depression among Dutch adolescents. Pediatrics. 2006;117:412-6.

14. Wedderkopp N, Leboeuf-Yde C, Bo Andersen L, Froberg C, Steen HH. Back Pain in Children, No Association with Objectively Measured Level of Physical Activity. Spine. 2003;28(17):2019-24

15. Kokko S, Selänne H, Alanko L, Heinonen O, Korpelainen R, Savonen K, et al. Health promotion activities of sports clubs and coaches, and health and health behaviours in youth participating in sports clubs: the Health Promoting Sports Club study. BMJ Open Sport Exerc Med. 2015;1:e000034.

16. Currie C, Aleman-Diaz A. Building knowledge on adolescent health: reflections on the contribution of the Health Behaviour in School-aged Children (HBSC) study. Eur J Pub Health. 2015;25(Supp 2):4-6.

17. Alaranta A. Medication use in Elite Athletes. University of Helsinki. Yliopistopaino: Helsinki. 2006

18. Kokko S. Health Promoting Sports Club - Youth Sports Clubs' Health Promotion Profiles, Guidance, and Associated Coaching Practice, in Finland. University of Jyväskylä, Studies in Sport, Physical Activity and Health 144. 2010.

19. Kokko S, Kannas L, Villberg J, Ormshaw M. Health promotion guidance activity of youth sports clubs. Health Education. 2011a; 111:452-63.

20. Pasanen $\mathrm{K}$, Parkkari J, Pasanen $\mathrm{M}$, Hiilloskorpi H, Mäkinen T, Järvinen $\mathrm{M}_{\text {, }}$ Kannus $P$. Neuromuscular training and the risk of leg injuries in female floorball players: cluster randomised controlled study. BMJ. 2008;337:96-102.

21. Kuorinka I, Jonsson B, Kilbom A, Vinterberg H, Biering-Sørensen F, Andersson $G$, et al. Standardised Nordic questionnaires for the analysis of musculoskeletal symptoms. App Ergonomics. 1987;18:233-7.
22. Aira, T, Kannas, L, Tynjälä, J, Villberg, J, Kokko, S. Liikunta-aktiivisuuden väheneminen murrosiässä. Drop off-ilmiön aikatrendejä ja kansainvälistä vertailua WHO-Koululaistutkimuksen (HBSC-Study) aineistoilla 1986-2010. Miksi murrosikäinen luopuu liikunnasta? Valtion liikuntaneuvoston julkaisuja 3. [Diminishing Physical Activity in Adolescence. The Time Trends and International Comparisons of the Drop-off Phenomena in the 1986-2010 Data of Health Behavior in School-aged Children Study. In the publication Why adolescents give up on physical activity? Publications of State Council of Sport 3.] 2013. 2013, 12-29.

23. Kokko S. Sports clubs as settings for health promotion: Fundamentals and an overview to research. Scand J Public Healt. 2014; 42 Suppl 15: 60-65.

24. Kokko S, Kannas L, Villberg J. Health promotion profile of youth sports clubs in Finland: club officials' and coaches' perceptions. Health Promot. Int. 2009; 24:26-35.

25. Liukkonen J, Jaakkola T, Kokko S, Gråstén A, Yli-Piipari S, Koski P, et al. Results from Finland's 2014 Report Card on Physical Activity for Children and Youth. J Phys Act Health. 2014;11(Supp. 1):51-7.

26. Hakkarainen $H$, Härkönen A, Niemi-Nikkola K, Mäenpää P, Potinkara P, Kujala A et al. (eds.) Selvitysraportti - Urheilevien lasten ja nuorten fyysismotorinen harjoittelu. Nuori Suomi ry, Suomen Olympiakomitea ry, Suomen Valmentajat ry. [Report - The physio-motor training of children and adolescents. The Young Finland assoc., The Finnish Olympic Committee, The Coaching assoc. Finland]. Helsinki: SLU-paino. 2008.

27. Eiosdottir SP, Kristjansson AL, Sigfusdottir ID, Allegrante JP. Trends in physical activity and participation in sports clubs among Icelandic adolescents. Eur J Public Health. 2008:18:289-93.

28. Kokko S, Villberg J, Kannas L. Nuori urheilijan polulla - 13 - 15 -vuotiaiden urheilijoiden arvioita harjoitus-määristään, harjoittelun monipuolisuudesta sekä elämäntavoista. Nuoren Suomen julkaisuja. [Youth on the athlete track - the evaluation of 13 to 15 years old athletes on their training volume, versatility and lifestyle. Publication of the Young Finland Association.] 2011c.

29. Harreby M, Nygaard B, Jessen T, et al. Risk factors for low back pain in a cohort of 1389 Danish school children: An epidemiologic study. Eur Spine J. 1999:8(6):444-50.

30. Van Gent C, Dols JJ, de Rover CM, Hira Sing RA, de Vet HC. The weight of schoolbags and the occurrence of neck, shoulder, and back pain in young adolescents. Spine (Phila Pa 1976). 2003;28(9):916-21.

31. Myrtveit SM, Sivertsen B, Skogen JC, Frostholm L, Stormark KM, Hysing M. Adolescent Neck and Shoulder Pain The Association With Depression, Physical Activity, Screen-Based Activities, and Use of Health Care Services. J Adolesc Health. 2014;55:366-72.

32. Jones MA, Stratton G, Reilly T, Unnithan VB. A school-based survey of recurrent non-specific low-back pain prevalence and consequences in children. Health Educ Res. 2004;19(3):284-9.

33. Hangai M, Kaneoka K, Okubo YPT, et al. Relationship between low back pain and competitive sports activities during youth. Am J Sports Med. 2010;38(4):791-6.

34. Mogensen AM, Gausel AM, Wedderkopp N, Kjaer P, Leboeuf-Yde C. Is active participation in specific sport activities linked with back pain? Scand J Med Sci Sports. 2007;17(6):680-6.

35. Ehrmann Feldman D, Shrier I, Rossignol M, Abenhaim L. Risk Factors for the Development of Neck and Upper Limb Pain in Adolescents. Spine. 2002;27:523-8.

36. Wedderkopp N, Kjaer P, Hestbaek L, Korsholm L, Leboeuf-Yde C. High-level physical activity in childhood seems to protect against low back pain in early adolescence. Spine J. 2009;9:134-41.

37. Østerås $N$, Ljunggren $A E$, Gould KS, Wærsted M, Veiersted KB. Muscle pain, physical activity, self-efficacy and relaxation ability in adolescents. Adv Physiother. 2006;8:33-40.

38. Mikkelsson LO, Nupponen H, Kaprio J, Kautiainen H, Mikkelsson M, Kujala UM. Adolescent flexibility, endurance strength, and physical activity as predictors of adult tension neck, low back pain, and knee injury: a 25 year follow up study. Br J Sports Med. 2006;40:107-13.

39. Taanila HP, Suni JH, Pihlajamaki HK, et al. Predictors of low back pain in physically active conscripts with special emphasis on muscular fitness. Spine J. 2012;12(9):737-48.

40. Kujala UM, Taimela S, Erkintalo M, Salminen JJ, Kaprio J. Low-back pain in adolescent athletes. Med Sci Sports Exerc. 1996;28:165-70.

41. Haydt R, Pheasant $S$, Lawrence K. The incidence of low back pain in NCAA division iii female field hockey players. Int J Sports Phys Ther. 2012;7(3):296305. 
42. Verhagen EALM, Van der Beek AJ, Bouter LM, Bahr RM, Mechelen WV. A one season prospective cohort study of volleyball injuries. Br J Sports Med. 2004;38(4):477-81.

43. Pasanen K, Rossi M, Parkkari J, Kannus P, Heinonen A, Tokola K, Myklebust G. Low back pain in young basketball and floorball players: a retrospective study. Clin J Sports Med. 2015:0:1-5.

44. Vikat A, Rimpelä M, Salminen JJ, Rimpelä A, Savolainen A, Virtanen SM. Neck or shoulder pain and low back pain in Finnish adolescents. Scand J Public Health. 2000;28(3):164-73.

45. Schmidt CP, Zwingenberger S, Walther A, Reuter U, Kasten P, Seifert J, Günther KP, Stiehler M. Prevalence of Low Back Pain in Adolescent Athletes - an Epidemiological Investigation. Int J Sports Med. 2014;35(8):684-9.

46. Ristolainen L, Kettunen JA, Waller B, Heinonen A, Kujala UM. Training-related risk factors in the etiology of overuse injuries in endurance sports. J Sports Med Phys Fitness. 2014;54(1):78-87.

47. Watson KD, Papageorgiou AC, Jones GT, Taylor S, Symmons DP, Silman AJ, Macfarlane GJ. Low back pain in schoolchildren: the role of mechanical and psychosocial factors. Arch Dis Child. 2003;88(1):12-7.

48. Maras D, Flament M, Murray M, Buchholz A, Henderson K, Obeid N, Goldfield G. Screen time is associated with depression and anxiety in Canadian youth. Prev Med. 2015;73:133-8.

\section{Submit your next manuscript to BioMed Central} and we will help you at every step:

- We accept pre-submission inquiries

- Our selector tool helps you to find the most relevant journal

- We provide round the clock customer support

- Convenient online submission

- Thorough peer review

- Inclusion in PubMed and all major indexing services

- Maximum visibility for your research

Submit your manuscript at www.biomedcentral.com/submit 